in xenotransplantation. We should also assume that viral replication and sustained viremia in humans as a result of nonhuman primate to human xenotransplantation could lead to disease-to knowingly introduce persistent viral infections such as SFV into humans via transplantation is never a sensible public health strategy in averting global pandemics.

JoNATHAN S. AlLAN

Dept. of Virology and Immunology

Southwest Foundation for Biomedical Research

7620 N.W. Loop 410 at Military Drive

San Antonio, Texas 78228

email: jallan@icarus.sfbr.org

Heneine and Chapman reply-Allan reasons that SFV, like SIV, may vary in infectivity and virulence for humans by species origin, and notes that macaques are the most common captive non-human primate (NHP) species, implying that the predominance of baboon-type SFV among the infected workers we reported may reflect increased infectivity or virulence of baboon-type SFV for humans. While SFV strains may vary in infectivity as argued, other factors explain the predominance of baboon-type SFV among our workers. Two of the three workers with baboon-type SFV were disproportionately exposed to baboons. Also, workers may be relatively protected against infection with macaque type SFV because stricter safety precautions taken to minimize risk of exposure to cercopithecine herpesvirus 1 (B virus), which is deadly for humans, simultaneously decrease exposure to SFV. Therefore, our findings do not support speculation on species-specific differences in SFVs.

In our article ${ }^{1}$, we discussed the issue of SFV transmission through infected blood and, as stated, we are counseling all SFVinfected persons to refrain from blood and tissue donation until risks of transmission are defined. The broader policy concerns raised by Allan are beyond the scope of our paper and are, we think, more appropriately addressed in other forums.

Our paper recognizes that prospective studies of larger numbers of infected persons occupationally exposed to NHPs are needed to clarify the full pathogenic potential and human-to-human transmissibility of SFV. We caution against interpreting our findings to either reassure or alarm beyond what our data can support.

Walid Heneine \& Louisa E. ChapMan HIV and Retrovirology Branch

Centers for Disease Control and Prevention

1600 Clifton Road, G-19

Atlanta, GA 30333, USA

email:WMH2@cdc.gov

1. Heneine, W. et al. Identification of a human population infected with simian foamy viruses. Nature Med. 4, 4030-407 (1998).

2. Broussard, S. R. et al. Characterization of new simian foamy viruses (SFV) from African nonhuman primates. Virology 237, 349-359 (1997).

3. Hirsch, V. M. \& Johnson, P. R. Pathogenic diversity of simian immunodeficiency viruses. Virus Res. 32, 183-203 (1994).

4. Kondo, T. et al. Risk of adult T-cell leukaemia/lym phoma in HTLV-I carriers [letter]. Lancet 2, 159 (1987)

5. Allan, I.S. et al. Amplification of simian retroviral sequences from human recipients of baboon liver transplants. AIDS Res. Hum. Retrovir 14, 819-822 (1998).

\section{External stenting \& atherosclerosis}

In the February issue, Mehta and colleagues' reported that the application of an external stent to a pig saphenous vein graft reduced medial thickening, intima formation and cell proliferation associated with reduced PDGF expression. As the study was conducted on normolipidemic animals, no conclusion relevant to preventing atherosclerosis was possible. Working on rabbits, we had previously reported that a rigid external stent applied to grafted jugular veins to prevent vein distention reduced the thickness and total cross-sectional area of the graft ${ }^{2}$. We also demonstrated that in hypercholesterolemic rabbits, rigid external stenting markedly reduced graft susceptibility to atherosclerosis $^{2}$. We believe that external stenting should be explored further as a means of preventing long-term failure of coronary bypass grafts due to atherosclerosis.

Alain Tedgui, Ziad Mallat \& STÉPHANIE LEHOUX

INSERM U141

Hôpital Lariboisière, 41 Boulevard de la Chapelle, 75475 Paris, Cedex 10, France

1. Mehta, D. et al. External stenting reduces longterm medial and neointimal thickening and platelet derived growth factor expression in a pig model of arteriovenous bypass grafting. Nature Med. 4, 235-239 (1998)

2. Batellier, J et al. Protection from atherosclerosis in vein graft by rigid external support. Arterioscler. Thromb. 13, 379-84 (1993).

\title{
Hypocretins or hyporexins?
}

To the editor-Recently Schwartz ${ }^{1}$ reviewed the importance of two new hypothalamic peptides, orexin A, 33 amino acids, and orexin B, 28 amino acids, in energy homeostasis. Molecular genetic studies in rodents have provided convincing evidence supporting relevant roles for several hypothalamic neuropeptides in the central regulation of energy balance. While $\alpha$-melanocyte stimulating hormone, glucagon-like peptide-1 and bombesin, which are positively regulated by leptin, have been established as potent negative regulators of feeding, neuropeptide $\mathrm{Y}$, galanin, opioids, melanin concentrating hormone and Agouti-related protein, which are negatively regulated by leptin, have been established as potent positive regulators of feeding behavior ${ }^{2}$. Of the late arrivals, the orexins have been characterized as potent stimulants of food intake ${ }^{3}$. Recently, De Lecea et al. ${ }^{4}$ described two hypothalamus-specific peptides with neuroexcitatory activity that share substantial amino acid identities with members of a family of hormones, the incretins, thus termed hypocretins. Even if not completely characterized from a functional point of view, the mRNA sequences (Mus musculus preprohypocretin cDNA accession number is AF019566), chromosomal mapping, and the cerebral immunolocalizations of these peptides identify them with orexin $\mathrm{A}$ and B (Mus musculus prepro-orexin CDNA accession number is AFO41242). Thus, would it be better to speak of hyporexins?

EnZo Nisoli \& Michele O. CarRUba
Center for Study and Research on Obesity LITA

Vialba L. Sacco Hospital

University of Milan, Milan, Italy 20157

csro@unimi.it

\section{Alessandra VALERIO}

Department of Biomedical Sciences and Biotechnologies University of Brescia

Brescia, Italy 25123

\section{GIUSEPPE BORSANI}

Tigem - Telethon Institute of Genetics and Medicine

Milan, Italy 20132

1. Schwartz, M.W. Orexins and appetite: the big picture of energy homeostasis gets a little bigger. Nature Med. 4, 385-386 (1998).

2. Flier, J.S. \& Maratos-Flier, E. Obesity and the hypothalamus: novel peptides for new pathways. Cell 92, 477-440 (1998).

3. Sakurai, T. et al. Orexins and orexin receptors: a family of hypothalamic neuropeptides and $G$ protein-coupled receptors that regulate feeding behavior. Cell 92, 573-585 (1998).

4. De Lecea, L. et al. The hypocretins: hypothalamus-specific peptides with neuroexcitatory activity. Proc. Natl. Acad. Sci. USA 95, 322-327 (1998). 